\title{
Restriction Endonuclease
}

National Cancer Institute

\section{Source}

National Cancer Institute. Restriction Endonuclease. NCI Thesaurus. Code C16515.

Enzymes that exhibit endonuclease activity at a specific short nucleotide sequence to cleave strands of DNA. 